\title{
Diabetic Foot Ulcers: A Prospective Study Of 100 Patients Based On Wound Based Severity Score.
}

\author{
Dr. Mohit Sharma ${ }^{1}$, Dr. Anil Sharma ${ }^{2}$, Dr. Sita Ram Gothwal ${ }^{3}$, Dr. Sunil Dixit ${ }^{4}$, \\ Dr. Ashok Kumar Lunia ${ }^{5}$, Dr. Mahima Sharma ${ }^{6}$ \\ 1. M.B.B.S., M.S., M.ch. (Resident) Department of Cardio-Vascular and thoracic surgery S.M.S. Medical \\ College, Jaipur ( Rajasthan) \\ 2. M.B.B.S., M.S., M.ch. Professor and Unit HeadDepartment of Cardio-Vascular and thoracic surgery; S.M.S. \\ Medical College, Jaipur (Rajasthan) \\ 3. M.B.B.S., M.S. Professor and Unit Head Department of General Surgery S.P.Medical College and P.B.M. \\ group of hospitals, Bikaner(Rajasthan) \\ 4. M.B.B.S., M.S, M.ch. Assistant Professor Department of Cardio-Vascular and thoracic surgery; S.M.S. \\ Medical College, Jaipur \\ 5. Assistant Professor M.B.B.S., M.S., Department of General Surgery S.P.Medical College and P.B.M. group \\ of hospitals, Bikaner(Rajasthan) \\ 6. M.B.B.S., M.D. Senior Demonstrator, Department of Physiology, Govt. Dental College, Jaipur
}

\begin{abstract}
The Diabetic foot is the commonest complication of Diabetes and is a leading cause of Hospitalization and prolonged in - patient treatment. Diabetic foot ulcer is far and away the most frequent indication for non traumatic lower limb amputations. Appropriate preventive measures as well as patient education will markedly reduce ulcer formation and the frequency of amputations in addition to cutting down on healthcare costs. Here we studied 100 diabetic patients on the basis of new wound based severity scoring system given by Beckert et al.
\end{abstract}

AIMS \& OBJECTIVES

1. Reviewing the established wound based clinical scoring system for diabetic foot ulcers suitable for daily clinical practice anticipating changes for healing and risk of amputation.

2. To evaluate the cause that leads to amputation \& develop methods to minimized the chances amputation.

3. Establishing awareness for diabetic foot and ulcers in diabetic patients.

\section{MATERIAL AND METHODS}

The study was conducted in the Department of General Surgery in collaboration with diabetic clinic, P.B.M. Hospital, Bikaner (Rajasthan) and S.M.S. Medical College, Jaipur (Rajasthan) from September 2010 to December 2013 in 100 patients.

For clinically defined parameters, namely palpable pedal pulses, probing to bone, ulcer location, and presence of multiple ulcerations, were prospectively assessed in 100 consecutive patients on the basis of new diabetic ulcer severity score (DUSS). Probability of healing and risk of amputation was calculated by the Kaplan Meier method.

\section{RESULTS AND CONCLUSIONS}

We conclude that DUSS provides an easy prognostic tool for anticipating probability of healing, hospital admission \& need for surgery by combining four clinically assembled wound based parameters and it is easy to apply DUSS in daily clinical practice.

Key Words: Diabetic foot, Ulcer, Amputation, Diabetic Ulcer Severity Score

\section{Introduction}

The World Health Organization (WHO) defines diabetic foot as the lower limb of a diabetic patient that has the potential risk of pathologic consequences, including infection, ulceration and/or destruction of deep tissues associated with neurological abnormalities, various degrees of peripheral vascular disease and/or metabolic complications of diabetes. Peripheral neuropathy, vasculopathy and superimposed infection are mainly responsible for diabetic foot ulceration. Ulcers which develop are difficult to treat due to poor wound healing which results from a combination of neuropathy, ischemia and hyperglycemia.

As at 2000, about 177 million persons were afflicted by diabetes which the WHO has predicted will rise to 300 million by 2025 [1]. Fifteen percent of diabetics develop foot ulcers during their life time with significant health related decrease in quality of life and consumption of a great deal of healthcare resources, 
Diabetic Foot Ulcers: A Prospective Study Of 100 Patients Based On Wound Based Severity Score.

while diabetic foot ulcers account for between $50 \%$ to $80 \%$ of non traumatic amputations $[2,3,4]$. Presently substantial progress is being achieved in the treatment of diabetic foot ulcers in centers that base their management on the twin pillars of preventive measures / patient education and a multi- disciplinary team approach from the onset.

\section{RISK FACTORS FOR DIABETIC FOOT ULCERATION [6]}

1. Peripheral neuropathy: somatic and autonomic

2. Peripheral vascular disease

3. Previous foot ulcers

4. Foot deformities: claw foot, charcot arthropathy

5. Presence of callus

6. Trauma

7. Nephropathy

8. Elderly if living alone

9. Poor understanding of diabetes

\section{Pathogenesis}

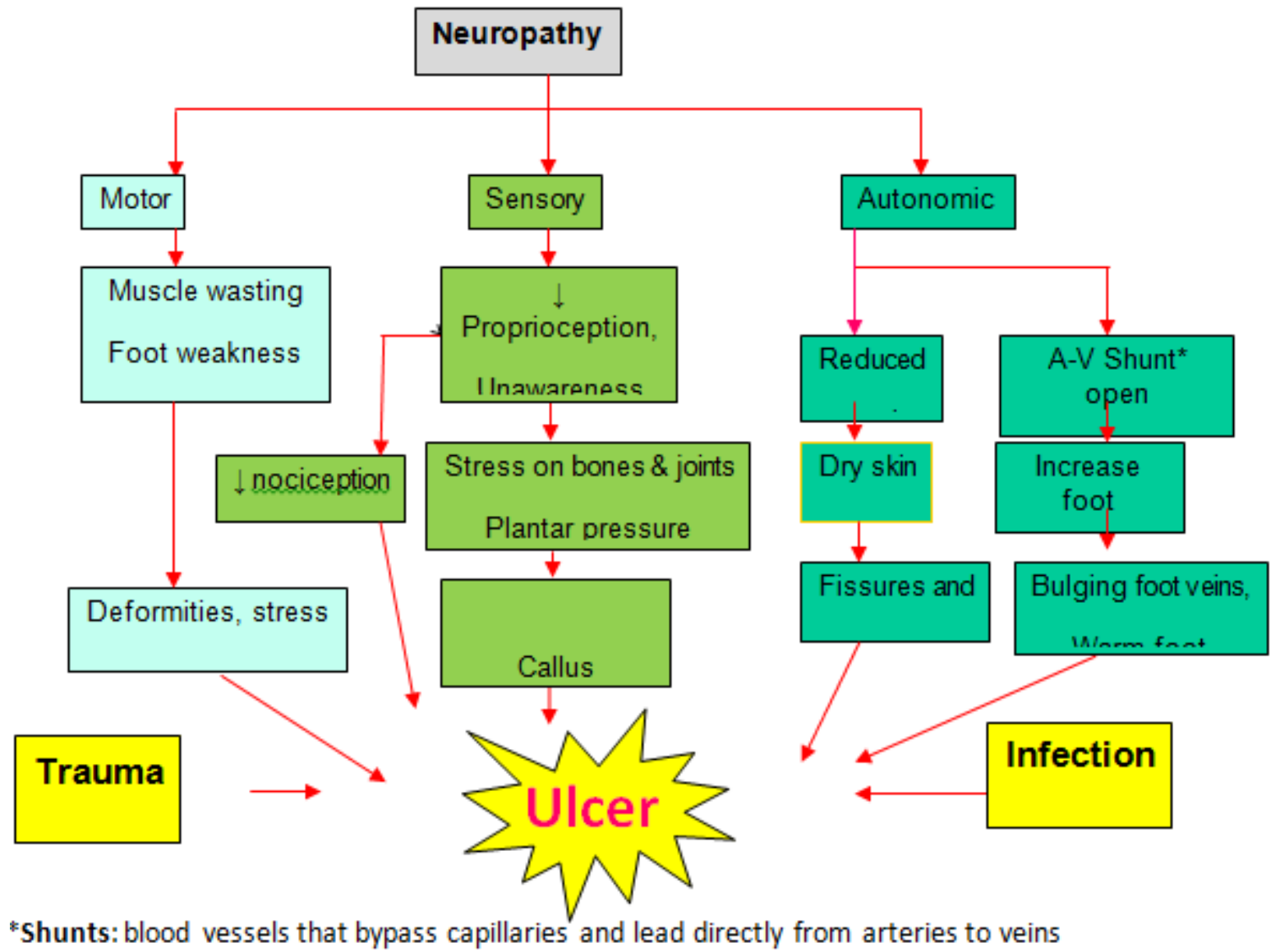




\section{Peripheral arterial disease}

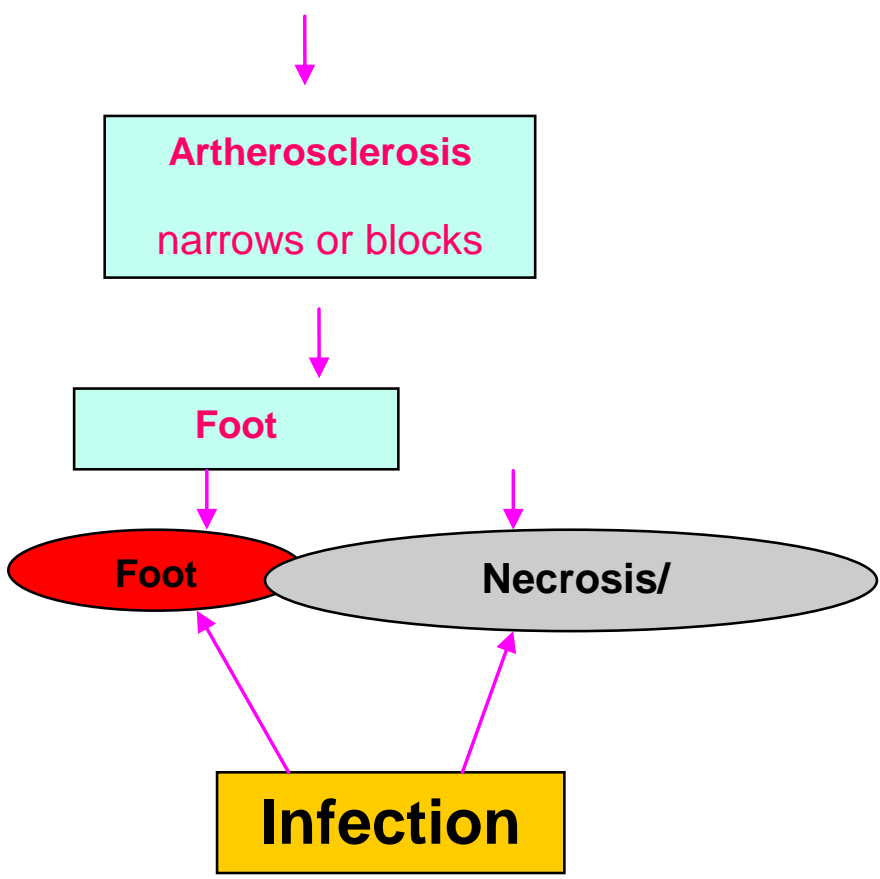

Since diabetic foot wounds and amputations account for a significant part of diabetes-related health care costs [5,6], several attempts have been made to establish classification systems that help assess the severity of disease. According to the International Working Group on the Diabetic Foot, a classification system appropriate for clinical practice should facilitate communication between health care providers, influence daily management, and provide information about the healing potential of an ulcer [7]. In 1976, Meggitt [8] described one of the most commonly cited wound classification systems that were further popularized by Wagner [9] in 1981. However, the Meggitt-Wagner classification exclusively assessed ulcer depth without comorbidities such as ischemia or pressure load [5]. More recently, the University of Texas system improved ulcer classification by including the parameters ischemia and infection [10,11]. A classification system using simple clinical methods was recently published by Treece et al. [12] consisting of the five parameters ulcer area, ulcer depth, sepsis, arteriopathy, and denervation.

In our study, we categorized diabetic foot ulcers according DUSS ( Diabetic Ulcer Severity Score) as described by Beckert et al, ranging from 0 to 4 using four wound-based clinical parameters: palpable pedal pulses, probing to bone, ulcer location (foot or toe ulcer), and the presence of multiple ulcers [13]. The aim of this study was to describe the impact of this scoring system on prediction of clinical outcome defined as probability of healing and risk for amputation.

\section{DIABETIC ULCER SEVERITY SCORE (DUSS)[13]}

In our prospective study these clinical parameters to assess the severity of disease in diabetic foot.

\begin{tabular}{|l|l|c|c|}
\hline \multicolumn{2}{|c|}{} & \multicolumn{2}{|c|}{ Scoring } \\
\cline { 3 - 4 } & \multicolumn{2}{|c|}{1} \\
\hline 2. & Palpable pedal pulses & Present & Absent \\
\hline 3. & Probing of bone & Absent & Present \\
\hline 4. & Ulcer location & Toe & Foot \\
\hline
\end{tabular}

Wagner classified diabetic foot lesions into various grades in order to guide the management. This classification is most widely used one [9]

Modified Wegner's Grading- 
Diabetic Foot Ulcers: A Prospective Study Of 100 Patients Based On Wound Based Severity Score.

\begin{tabular}{|l|l|}
\hline Grade & Lesion \\
\hline 0 & High risk foot, no ulcer \\
A. Ischemic B. Infected \\
\hline 1 & Superficial ulcer \\
A. Ischemic B. Infected \\
\hline 2 & $\begin{array}{c}\text { Deeper ulcer to tendon or joint capsule } \\
\text { A. Ischemic B. Infected }\end{array}$ \\
\hline 3 & $\begin{array}{c}\text { Deep ulcer with abscess, osteomyelitis or joint sepsis } \\
\text { A. Ischemic B. Infected }\end{array}$ \\
\hline 4 & Localized gangrene - forefoot or heel \\
& A. Ischemic B. Infected \\
\hline 5 & $\begin{array}{l}\text { Gangrene of whole foot } \\
\text { A. Ischemic B. Infected }\end{array}$ \\
\hline
\end{tabular}

\section{Study Design And Methods}

The study was conducted in the Department of General Surgery in collaboration with diabetic clinic, P.B.M. Hospital, Bikaner (Rajasthan) and S.M.S. Medical College, Jaipur (Rajasthan) from September 2010 to December 2013.

For clinically defined parameters, namely palpable pedal pulses, probing to bone, ulcer location, and presence of multiple ulcerations, were prospectively assessed in 100 consecutive patients on the basis of new diabetic ulcer severity score (DUSS). Palpable pedal pulses catergorized by the absence (scored as 1) or presence (scored as 0 ) of pedal pulses, while probing to bone was defined as yes (scored as 1) or no (scored as 0 ), the site of ulceration was defined as toe (scored as 0) or foot (scored as 1) ulcer. Patients with multiple ulcerations were graded as 1 compared with those with single ulcers (scored as 0). The DUSS was calculated by adding these separate grading to a theoretical maximum of 4 . Wounds were followed up 6-12 months or until healing or amputation if earlier. Probability of healing and risk of amputation was calculated by the Kaplan Meier method.

Selection of patients

A total of 100 patients suffering from foot ulcers in type 2 diabetes mellitus were selected randomly for the study, after explaining, informed written consent was taken before suitable procedure and after routine investigations.

Patient Population

(A) Inclusion criteria

1. Adult patients of any sex with D.M.

2. Clinical diagnosis of chronic and/or acute phase lower limb ulcers.

3. Ulcers located below ankle

(B) Exclusion criteria

1. An active neoplastic disease.

2. Immunosuppressive treatment in last three months.

3. Ulcers affecting above ankle.

4. Patients with less than two visits.

Patient Examination

Each selected subject underwent detailed history and complete physical examination. History regarding age, sex, socioeconomic status, rural or urban, duration of diabetes, treatment history of diabetes (i.e. diet therapy, insulin therapy, oral hypoglycemic agents only, oral hypoglycemic agents with insulin), family history of diabetes, and history of smoking and occupational history were taken.

The selected patients were evaluated for the presence of microvascular and macrovascular complications like coronary artery disease, cerebrovascular disease, peripheral vascular disease, retinopathy, nephropathy and neuropathy. Blood pressure was recorded in lying down, sitting and standing position at interval of five minutes and compared in both arms (at least three readings, five minutes apart were taken prior to labeling hypertension). Systemic hypertension was defined as systolic BP greater than or equal to $140 \mathrm{mmHg}$ and diastolic BP greater than or equal to $90 \mathrm{mmHg}$ or patients already on antihypertensive therapy, Peripheral vascular disease (PVD) was diagnosed by definitive history of intermittent claudication or if one or more peripheral pulses were absent in both feet and graded according to ankle brachial pressure index (ABPI) by Doppler and C.T. angio in department of radiology. Coronary artery disease was diagnosed by, history of angina or myocardial infarction or document by previous treatment records, pathological Q-wave in an ECG recording (Minnesota Codes), ST segment depression, $\mathrm{T}$ wave abnormalities, Chest X-ray to assess cardiac size. Neuropathy was diagnosed by history of numbness, paraesthesia, tingling sensation and burning sensation. It was confirmed by touch sensation by 1-0 monofilament and ankle reflex. A history of pain worsening at night helped in diagnosing painful peripheral neuropathy. Autonomic neuropathy was diagnosed by history of postural fall of blood pressure, 
history of constipation or diarrhea and gastroparesis. Fundus was examined in each patient to look for evidence of retinopathy by ophthalmoscope and if needed findings were confirmed by an ophthalmologist. Glycosylated hemoglobin $(\mathrm{GHb})$ provides fairly average blood glucose concentration over the preceding several weeks. Cerebrovascular disease was diagnosed by past history of CVA. Patients with monoparesis or hemiparesis or hemiplegia, sudden onset aphasia, facial weakness with other signs of cerebrovascular disease were clinically assessed and confirmed by computed tomography of head. Early Nephropathy was diagnosed in patients who had microalbuminuria (urinary albumin $>30 \mathrm{mg} / 24 \mathrm{hrs}$. but $<300 \mathrm{mg} / 24$ hrs.). Overt renal failure was diagnosed by macroalbuminuria (> 300mg of albumin in 24 hrs. collected urine) and elevated serum creatinine level. Lipid profile was done in every patient and abnormality if any, was detected and recorded. We also assessed the diabetic foot ulcer and/or gangrene by detailed clinical history and examination. as given in following protocol -

\section{Signs of ischemia}

Claudication time.

Colour of limb .

Appearance of toes

Hair distribution over digits and dorsum of foot

2. Features of ulcer

Location of ulcer

Shape \& size of ulcer

Edge \& margin of ulcer

Base \& floor of ulcer

Discharge from ulcer

Depth of ulcer

Surrounding skin

3. Arterial pulsations

Dorsalis pedis artery

Anterior \& posterior tibial arteries

Popletial artery

Femoral artery

4. Peripheral neuropathy

Touch

Ankle reflex

Vibrational sense

5. Ankle Brachial Pressure Index (ABPI)

The infected ulcers were swabbed, cultured and debrided and were dressed with either of the following: Saline gauze, Povidone-iodine, Hydrogel, Hydrocolloid based Salutyl (Collegenase), Calgigraph (Calcium Alginate), Plermin1 (Human Recombinant Platelet Derived Growth Factor). Antibiotics were prescribed as per culture and sensitivity report and response of the wound infection. Where the ulcers did not heal completely but were infection free and healthy red granulation tissue was present, split skin grafting was done. Patients with gangrene underwent appropriate amputations. Patients were followed up till discharge from surgical ward and were referred to Department of Medicine for glycemic control and follow up.

\section{OBSERVATIOS-}

TABLE A

Baseline demographic detail:

\begin{tabular}{ll}
\hline Details & \\
\hline Patients & M : 68, F: 32 \\
Sex & $70(36-93)$ \\
age (years) & $5.5(2-60)$ \\
Number of Visits & 40 \\
Multiple ulcers & $120(60-365)$ \\
Time of follow-up (days) & 62 \\
Hospitalization & $60(30-2190)$ \\
Wounds & $8(0.9-138)$ \\
Wound history (days) & 37 \\
Wound area (cm $\left.{ }^{2}\right)$ & 28 \\
Soft tissue infection at initial visit & toe 38, foot 62 \\
Probing to bone & 71 \\
Ulcer location & \\
Palpable peripheral pulses & 37 \\
Wound Grading & 28
\end{tabular}


Diabetic Foot Ulcers: A Prospective Study Of 100 Patients Based On Wound Based Severity Score.

$\begin{array}{ll}\text { Grade III } & 2 \\ \text { Grade IV } & 5 \\ \text { Grade V } & 28 \\ \text { Surgery } & 100 \\ \text { Sharp debridement } & 9 \\ \text { Bone resection } & 8 \\ \text { Minor amputation } & 6 \\ \text { Major amputation } & \end{array}$

TABLE B

Subgroup analysis with respect to ulcer severity score

\begin{tabular}{|c|c|c|c|c|c|}
\hline DUSS & Wounds $(\mathrm{n})$ & Wound Size $\left(\mathrm{cm}^{2}\right)$ & Wound Duration (days) & Surgery $(\%)$ & Hospitalization $(\%)$ \\
\hline 0 & 36 & $2(0.9-40)$ & $\begin{array}{c}33 \\
(31-230)\end{array}$ & 0 & 5.56 \\
\hline 1 & 23 & $8(1-45)$ & $60(45-76)$ & 0. & 95.62 \\
\hline 2 & 9 & $4(2-32)$ & $\begin{array}{c}58 \\
(31-200)\end{array}$ & $22 . .22$ & 77.77 \\
\hline 3 & 9 & $50(6-136)$ & $\begin{array}{c}190 \\
(92-1825)\end{array}$ & 77.78 & 100 \\
\hline 4 & 23 & $110(4-138)$ & $\begin{array}{c}1460 \\
(150-2190)\end{array}$ & 100.00 & 100 \\
\hline
\end{tabular}

TABLE -C

Distribution of patients according to DUSS v/s type of management

\begin{tabular}{|c|c|c|c|c|c|c|c|c|c|}
\hline \multirow{2}{*}{ DUSS } & \multirow{2}{*}{$\begin{array}{c}\text { No. of } \\
\text { Patients }\end{array}$} & \multirow{2}{*}{ Debridement } & \multicolumn{2}{|c|}{$\begin{array}{c}\text { Major } \\
\text { Amputation }\end{array}$} & \multicolumn{2}{|c|}{$\begin{array}{c}\text { Minor } \\
\text { Amputation }\end{array}$} & \multicolumn{2}{|c|}{ Bone Resection } & \multirow{2}{*}{$\begin{array}{c}\% \text { of } \\
\text { Patients } \\
\text { require } \\
\text { Surgery }\end{array}$} \\
\hline & & & No. & $\%$ & No. & $\%$ & No. & $\%$ & \\
\hline 0 & 36 & 36 & 0 & 0.00 & 0 & 0.00 & 0 & 0.00 & 0.00 \\
\hline I & 23 & 23 & 0 & 0.00 & 0 & 0.00 & 0 & 0.00 & 0.00 \\
\hline II & 9 & 9 & 0 & 0.00 & 2 & 22.22 & 0 & 0.00 & 22.22 \\
\hline III & 9 & 9 & 1 & 11.11 & 5 & 55.56 & 1 & 11.11 & 77.78 \\
\hline IV & 23 & 23 & 6 & 26.09 & 8 & 34.78 & 9 & 39.13 & 100.00 \\
\hline
\end{tabular}

In our study we studied factors that affect healing \& association with diabetic foot. We observed impact of these factors on healing. In a prospective trial of 1000 patients of Diabetic foot ulcer Beckert et al 2006 gave a wound based severity scoring system, known as DUSS (Diabetic Ulcer Severity Score) [13]. According to Beckert et al in Uni \& multivariate analysis a significantly higher probability of healing for patients with palpable pulses, no probing of bone, toe ulcers \& absence of multiple ulcerations. In this prospective study we observed the DUSS in our clinical setting. In total 100 patients 68 patients were male \& 32 patients were female. Median age of Patients 70 year, Median No. of visits 5.5, multiple ulcers were presented in 40 patients, median time of follow up 120 days \& patients were hospitalized. We observed the clinical parameters individually with the impact on healing/type of treatment.

The clinical parameters we studied were-

1. Wound history (days)

2. Wound Area $\left(\mathrm{cm}^{2}\right)$

3. Soft tissue infections at initial visit

4. Probing of bone

5. Ulcer location

6. Palpable pedal pulses

7. Wagner's wound grade.

8. Number of ulcers.

9. Time of follow up.

10. Number of Visits.

After observing all clinical parameters we compare them with type of treatment/management so we were able to get effect of these clinical parameters on impact on healing of wound. In our study we select 100 patients. 68 were Male \& 32 were Female, all 100 patients were treated with debridement \& surgically. In surgical procedure major amputation, minor amputation \& bone resection were done. 63 patients were hospitalized \& 37 were on OPD basis. In 63 hospitalized patients 31 patients were treated surgically \& in 37 non hospitalized patients 1 was treated with minor amputation. 
After taking all parameters we classified patients according to DUSS (Table-B and Table-C). In our study 36 patients were presented in score 0,23 patients were presented in score 1, 9 patients were presented in score II. 9 patients were presented in score III, 23 patients were presented in score IV. In score 0 all 36 patients were treated with debridement only. In score I all 23 patients were treated again debridement only. In score II 2 patients out of 9 patients were treated with minor amputation i.e. $20.63 \%$. In score III 1 patient treated with major amputation, 5 patient with minor amputation \& 1 patient was treated with bone resection i.e. 7 patients out of 9 patients i.e. $77.78 \%$ needed major surgical treatment. In score IV all 23 patients needed major surgical treatment i.e. $100 \%$ patients needed major surgical treatment. So increasing in DUSS is directly related to increasing the chances of major surgical treatment i.e. Major amputation, minor amputation \& bone resection.

\section{DISCUSSION AND CONCLUSION}

The spectrum of diabetic complications is very wide, and to an extent unpredictable. The life expectancy of diabetics with gangrene of foot has increased over the years. Advances in insulin therapy and anti-diabetic drugs have increased the patient compliance and a better control over blood sugar. Newer technology in electronic gadgets has led to reliable and minimally painful, daily monitoring of fasting blood sugar which is a key factor in the outcome of affected limb. Foot problems are the most common indication for admission in the surgical ward in diabetic population. They account for $20 \%$ of all hospital admissions among the diabetics. Approximately $50 \%$ of all non traumatic amputations occur in diabetics with foot complications.

The following conclusions were drawn from the present study:

1. Diabetic foot is seen most commonly in 6th decade of life and male patients are affected more than female patients.

2. Peripheral neuropathy is the most common accompaniment.

3. Almost half of the patients suffer from medium to small sized arterial disease as evidenced by loss of pulsation from popletial to dorsalis pedis artery.

4. Trauma whether noticed or unnoticed is the predisposing factor in nearly every case.

5. Forefoot is the most common site of ulceration in diabetic foot.

6. Higher the grade of ulcer according to DUSS Classification more the chances of amputation.

7. Early and aggressive treatment should be the aim of the treating surgeon, so as to sacrifice some part, to salvage the limb and it's function.

8. Patients' awareness and education is the single most important factor for the prevention of disability and reduction in loss of limb or life.

9. Establishing a new wound based severity score for diabetic foot that can provide a predictive prognosis of limb.

10. If we adopt the following strategy, we can minimize the chance of Amputation.

First \& foremost is the prevention of development of Diabetic foot.Early and Aggressive treatment based on multidisciplinary approach including surgeon, physician, Diabetologist, Microbiologist and Anaesthetist. Early thorough debridement and effective wound care.

\section{Foot Care:}

1. Wash your feet daily (use a mild soap and lukewarm water).

2. Dry very carefully especially between the toes. It often helps to use talcum powder to dust the foot to furthre reduce moisture, however be certain to remove all the powder after dusting, as it should not leave a residue between the toes. If the skin is dry, use a good emollient - but not between the toes.

3. Inspect your foot daily (check for sores, cuts, bruise, changes to the toenails; use a mirror to look under the foot if you can not see it).

4. Look after your health (lose weight; stop smoking; exercise; manage your sugar; reduce alcohol consumption).

5. Look after your feet: Cut toenails straight across and never cut into the comers; use an emery board or file on sharp corners.

6. Do not try to remove corns or callus yourself - see a Podiatrist for this; do not use commercial com cures - this is important in those with diabetes as it is so easy to damage the skin.

7. Avoid going barefoot; even in your own home, this lessens the chance of some accidental damage.

8. Fitting of footwear is very important. Poorly fitted shoes are a common cause of problems in the foot of those with diabetes. Some advice: Get your feet measured each time you buy new shoes (foot size and 
shape change over time). New shoes should be comfortable when purchased and should not need a "break-in" period.They should fit both the length and width of the foot, with plenty of room for the toes.

9. Avoid shoes with high heels, pointed toes or tight around the toes which can contribute to ulcers.

10. See a Podiatrist/ health care personal at least annually.

\section{References-}

[1]. The Projection of prevalence and cost in Diabetes in Canada 2000 to 2016. Canadian Journal of Diabetes 2004.

[2]. Pinzur MS. Diabetic Foot. Available at http://www.emedicine.com/orthoped/topic 387.htm Accessed 21/07/08.

[3]. Wieman TJ. Principles of Management: The Diabetic foot. American Journal of Surgery 2005; 190:295- 299.

[4]. Levin ME. An Overview of the Diabetic Foot: Pathogenesis, Management and Prevention of Lesions. Int J Diabetes. Dev Countries $1994 ; 14$.

[5]. Apelqvist J, Ragnarson-Tennvall G, Larsson J, Persson U: Long-term costs for foot ulcers in diabetic patients in a multidisciplinary setting. Foot Ankle Int 16:388- 394, 1995

[6]. Cutaneous Diabetic Complications in the Lower Extremities. At http://www.powerpak.com/courses/2857. Accessed 20/07/ 08.

[7]. Schaper NC: Diabetic foot ulcer classification system for research purposes: a progress report on criteria for including patients in research studies. Diabetes Metab Res Rev 20 Suppl 1:S90 -95, 2004.

[8]. Meggitt B: Surgical management of the diabetic foot. Br J Hosp Med 16:227-232, 1976.

[9]. Wagner FW: The dysvascular foot: a system for diagnosis and treatment. Foot Ankle Clin 2:64 -122, 1981.

[10]. Lavery LA, Armstrong DG, Harkless LB: Classification of diabetic foot wounds. J Foot Ankle Surg 35:528 -531, 1996.

[11]. Armstrong DG, Lavery LA, Harkless LB: Validation of a diabetic wound classification system. Diabetes Care 21:855- 859, 1998.

[12]. Treece KA, Macfarlane RM, Pound N, Game FL, Jeffcoate WJ: Validation of a system of foot ulcer classification in diabetes mellitus. Diabet Med 21:987-991, 2004

[13]. Beckert et al. (2006) a new wound based severity score for diabetic foot ulcers: a prospective analysis of 1000 patients. Diabetic care 29: 988-992.

[14]. Lipsky BA, Sheehan P, Armstrong DG, Tice AD, Polis AB, Abramson MA. Clinical predictors of treatment failure for diabetic foot infections: Data from a prospective trial. Int wound J 2007; 4:30-38.

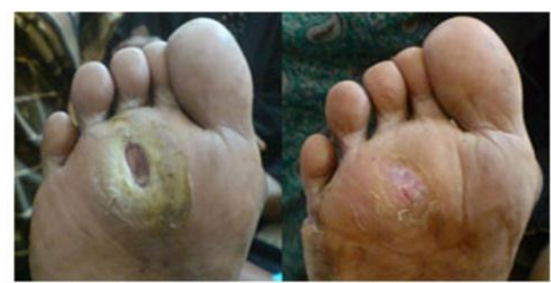

DUSS - I ( Single ulcer present on foot, with palpable pulses, no bone probing )

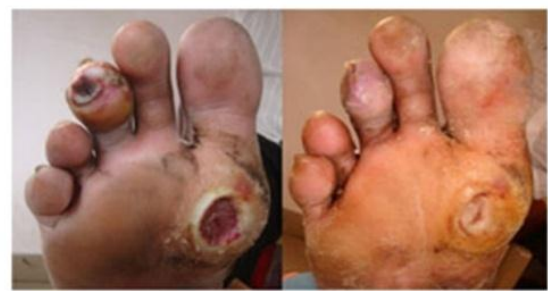

DUSS - I ( Single ulcer present on foot, with palpable pulses, no bone probing )

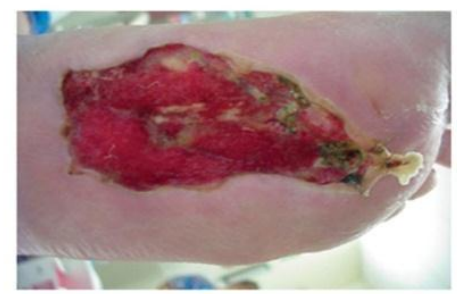

DUSS - I (Single ulcer on foot with no bone probing and palpable peripheral pulses) 


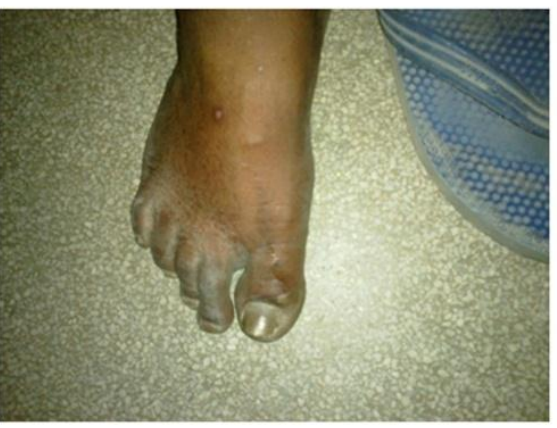

DUSS - II ( Single toe ulcer, non palpable pulses Pre-op)

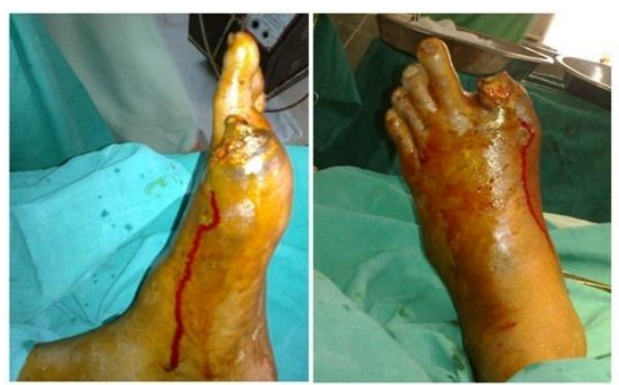

(Post - op)

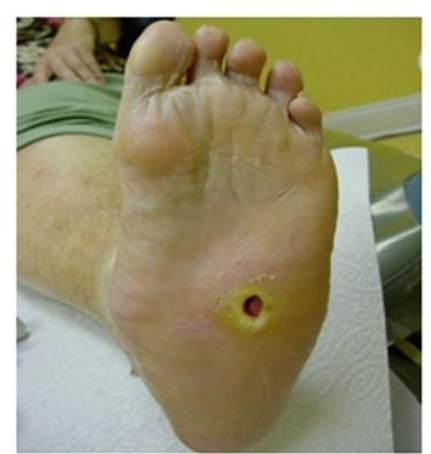

DUSS - II (Single foot ulcer with involvement of bone) 


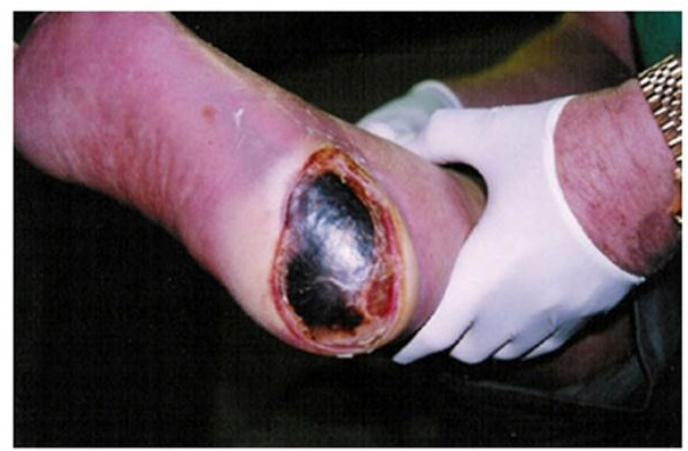

DUSS - III ( Ulcer Present on heel with bone probing present with absent pulses )

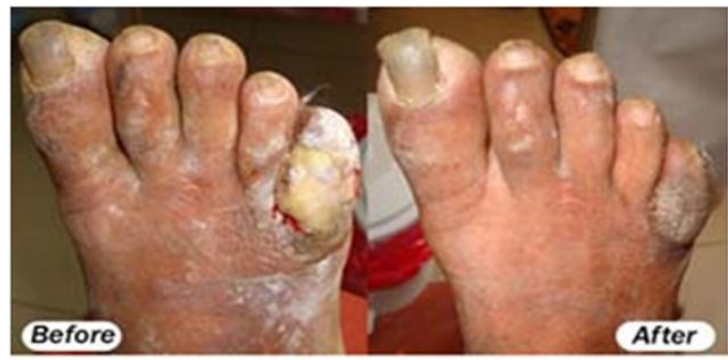

DUSS - 0 (Single ulcer on toe)

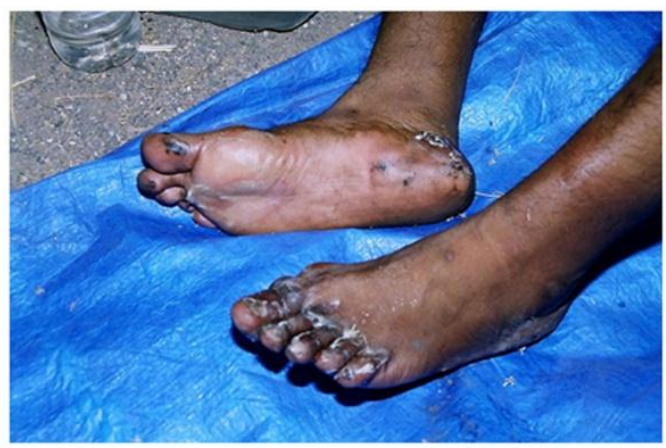

DUSS - III ( Multiple ulcers involving toe and foot with no palpable pulses) 


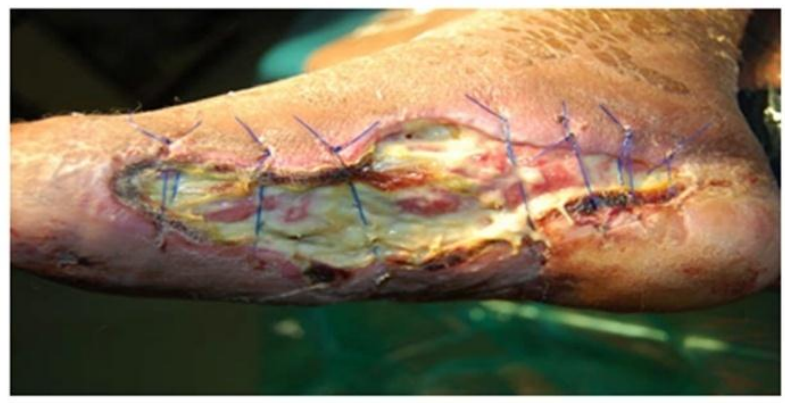

DUSS - IV ( Ulcer involving whole foot, bone probing present with no palpable pulses)

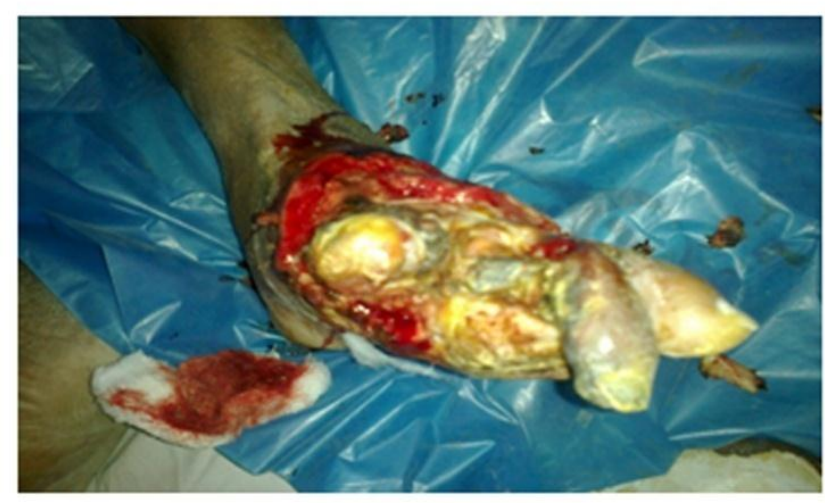

DUSS - IV ( Ulcer involving whole foot with bone probing with no palpable pulses) 